TRANSACTIONS OF THE

AMERICAN MATHEMATICAL SOCIETY

Volume 211,1975

\title{
SEMIFREE ACTIONS ON HOMOTOPY SPHERES
}

\author{
BY
}

\section{KAI WANG}

ABSTRACT. In this paper, we study the semifree $Z_{m}$ actions on homotopy sphere pairs. We show that in some cases the equivariant normal bundle to the fixed point set is equivariantly stably trivial. We compute the rank of the torsion free part of the group of semifree actions on homotopy sphere pairs in some cases. We also show that there exist infinitely many semifree $Z_{4 s}$ actions on even dimensional homotopy sphere pairs.

0. Introduction. Let $G$ be a compact Lie group. A differentiable group action of $G$ on a differentiable manifold $M^{n}$ is a homomorphism $\psi: G \rightarrow$ $\operatorname{Diff}(M)$, where $\operatorname{Diff}(M)$ is the group of diffeomorphisms of $M$. Let $F^{k}$ be the submanifold of fixed points. A group action is semifree if the only isotropy subgroups are the trivial subgroup and the group itself. Under these restrictions, the action is linear in some neighborhood of $F^{k}$ in $M^{n}$ in the sense that there is an equivariant vector bundle $\nu$ normal to $F^{k}$ in $M^{n}$ such that the action restricts on each fiber of $\nu$ to a linear automorphism. In this paper, we are only interested in the differentiable semifree actions on homotopy sphere pairs, namely, the actions on homotopy spheres such that the fixed point sets are homotopy spheres. The action $G=S^{1}$ defines a complex structure on $\nu$ (the action on $\nu$ is just that induced by the complex multiplication). In [2], Browder proved that for a semifree $S^{1}$ action on a homotopy sphere pair, the normal bundle of the fixed point set is stably trivial as a complex vector bundle. The first question which interests us is the following:

Problem 1. Is it true that the equivariant normal bundle to the fixed point set of a semifree $Z_{m}$ action on a homotopy sphere pair is equivariantly stably trivial?

If $G=S^{1}$ or $Z_{m}$, it is known (see [2], [6]) that there are infinitely many semifree $G$ actions on odd dimensional homotopy sphere pairs. As to semifree $S^{1}$ actions on even dimensional homotopy sphere pairs, there are only finitely many [3].

Received by the editors February 22, 1974 and, in revised form, July 9, 1974. AMS (MOS) subject clas sifications (1970). Primary 57E25, 57E30.

Key words and phrases. G-signatures, semifree actions, equivariant normal bundles, Chern classes. 
Problem 2. Is it ture that there are only finitely many semifree actions of $Z_{m}$ on even dimensional homotopy sphere pairs?

There is a natural group structure on the set of semifree actions on the homotopy sphere pairs with the same local representation. The classification scheme has been given by Browder and Petrie [3] and Rothenberg [5] Through use of this scheme the rank of the torsion free part of the group of semifree $S^{1}$ actions $\left(Z_{2}\right.$ actions, respectively) on homotopy sphere pairs has been computed in [3].

Problem 3. Calculate the rank of the torsion free part of the group of semifree $Z_{m}$ actions on homotopy sphere pairs.

It turns out that these three problems are related by the exact sequence of Rothenberg $($ see $\$ 1)$. We show that if $m \neq 0(\bmod 4)$, then, at least for a large family of $Z_{m}$ actions on homotopy sphere pairs, the answer to Problem 1 is positive. This, in turn, yields solutions to Problems 2 and 3. As for the case $m \equiv 0(\bmod 4)$, the answers to Problems 1 and 2 are, in general, negative; but we still are able to solve Problem 3, at least for a large family of groups of $Z_{m}$ actions on homotopy sphere pairs. These solutions are the main results of this paper.

This paper is organized as follows: In $\$ 1$, we describe the geometric situation, and we quote the results of Browder, Petrie, and Rothenberg. In $\$ 2$, we will show how to compute the determinant of some matrices that occur in the $G$-signature formula. In $\$ 3$, we study the functions $\Phi_{k}(\theta)$ which occur as coefficients in the $G$-signature formula. In $\$ 4$, we prove our main results.

I would like to thank Professor R. Schultz for providing me the estimation of when det $\widetilde{\Phi}_{r} \neq 0[8]$, the referee for several valuable suggestions, and Professor N. D. Kazarinoff for reading the manuscript.

1. Known results. Let $G$ be a compact Lie group. $A$-manifold $M^{n}$ is a manifold with a fixed $G$ action on it. Let $F^{k}$ be the submanifold of fixed points. The action is semifree if it acts freely on the complement of $F^{k}$. Let $\nu$ be the $G$-equivariant normal bundle to $F^{k}$ in $M^{n}$. The action on each fiber of $\nu$ is linear and represents an $(n-k)$-dimensional representation $\rho$ of $G$. The conjugacy class of this representation is constant on the connected component of $F^{k}$. We always assume that $F^{k}$ is simply-connected. Let $C(G, \rho)$ be the centralizer of $\rho(G)$ in $O(n-k)$. Then there is a reduction of the structural group of the bundle $\nu$ from $O(n-k)$ to $C(G, \rho)$, and this in turn induces a reduction of the group of $T M \mid F^{k}$ to the connected component 
of the identity of $C(G, \rho) \times O(k)$. Note that if $F^{k}$ is simply-connected, such a reduction always exists. $A(G, \rho)$-manifold $M^{n}$ is a $G$-manifold such that the local representation is equivalent to $\rho$ and which is furnished with a specific reduction of the group of $T M \mid F^{k}$ to the identity component of $C(G, \rho)$ $\times O(k)$. Two $(G, \rho)$-manifolds $M^{n}, N^{n}$ are equivalent if there is a $G$-equivariant diffeomorphism $f: M^{n} \rightarrow N^{n}$ which preserves the $(G, \rho)$-orientation. On the set of equivalence classes of $(G, \rho)$-manifolds we can define a $(G, \rho)$ oriented connected sum in a manner formally the same as that for the ordinary oriented connected sum. With this in mind, let $\mathcal{S}^{n}(G, \rho)$ be the set of equivalence classes of semifree $(G, \rho)$-manifolds $M^{n}$ such that $M^{n}$ and $F^{k}$ are homotopy spheres.

Proposition 1.1 [6]. For $k \geq 1, S^{n}(G, \rho)$ under the $(G, \rho)$-oriented connected sum is an abelian group.

Let $a_{1}$ and $a_{2}$ represent elements of $\mathcal{S}^{n}(G, \rho)$. We say $a_{1}$ is $h$-cobordant to $a_{2}$ if there is a semifree $(G, \rho)$-manifold $W^{n+1}$ which is homotopy equivalent to $S^{n} \times[0,1]$ and $F(G ; W)$ is homotopy equivalent to $S^{k} \times[0,1]$ in such a way that $\partial_{i} W(i=1,2)$ as a $(G, \rho)$-manifold is equivalent to $a_{i}$. Let $\mathcal{C}^{n}(G, \rho)$ be the set of $h$-cobordism classes of $\mathcal{S}^{n}(G, \rho)$. It is routine to check that the $(G, \rho)$-oriented connected sum preserves h-cobordisms, and hence $\mathcal{C}^{n}(G, \rho)$ is a quotient group of $\mathcal{S}^{n}(G, \rho)$. An element of $\bar{R}^{n}(G, \rho)$ is an equivalence class of objects, where an object is

(a) a $(G, \rho)$-oriented homotopy sphere $\Sigma^{n}$,

(b) a $(G, \rho)$-oriented imbedding $\psi: S^{k} \times R^{n-k} \rightarrow \Sigma^{n}$ such that $G$ acts freely on $\Sigma^{n}-\psi\left(S^{k} \times 0\right)$ and $G$ acts on $S^{k} \times R^{n-k}$ by $g(x, y)=(x, \rho(g) y)$. An equivalence of two objects $\left(\Sigma_{1}^{n}, \psi_{1}\right),\left(\Sigma_{2}^{n}, \psi_{2}\right)$ is an equivariant diffeomorphism $d: \Sigma_{1}^{n} \rightarrow \Sigma_{2}^{n}$ that preserves the $(G, \rho)$-orientation and such that $\psi_{2}=\psi_{1} \cdot d$. $\bar{R}^{n}(G, \rho)$ is a group under the $(G, \rho)$-oriented connected sum. Let $b_{i}(i=1,2)$ represent an element of $\bar{R}^{n}(G, \rho)$. We say $b_{1}$ is $h$-cobordant to $b_{2}$ is there is a $(G, \rho)$-manifold $W^{n+1}$ which is homotopy equivalent to $S^{n} \times[0,1]$ and a $(G, \rho)$-oriented imbedding $\psi: S^{k} \times[0,1] \times$ $R^{n-k} \rightarrow W^{n+1}$ such that $G$ acts freely on $W^{n+1}-\psi\left(S^{k} \times[0,1] \times 0\right)$ and $G$ acts on $S^{k} \times[0,1] \times R^{n-k}$ by $g(x, t, y)=(x, t, \rho(g) y)$ in such a way that $\partial_{i} W^{n+1}(i=1,2)$ as an element of $\bar{R}^{n}(G, \rho)$ is equivalent to $b_{i}$. Let $\Re^{n}(G, \rho)$ be the set of $h$-cobordism classes of $\bar{\Re}^{n}(G, \rho)$. It is easy to check that $R^{n}(G, \rho)$ is a group under the $(G, \rho)$-oriented connected sum. There is a map $\omega: \mathbb{R}^{n}(G, \rho) \rightarrow \mathcal{C}^{n}(G, \rho)$ defined in the obvious way. Let $\gamma: \mathcal{C}^{n}(G, \rho) \rightarrow \Gamma_{k}$ $+\pi_{k-1}(C(G, \rho))$ be defined as follows. Since the elements of $\pi_{k-1}(C(G, \rho))$ classify the equivariant bundles over $S^{k}$, the map $\gamma$ assigns to each element in $\mathcal{C}^{n}(G, \rho)$ its fixed point set and the equivariant normal bundle. 
Theorem 1.2 (Rothenberg [5]). The following sequence $\cdots \rightarrow \mathfrak{R}^{n}(G, \rho) \stackrel{\omega}{\longrightarrow} \mathcal{C}^{n}(G, \rho) \stackrel{\gamma}{\longrightarrow} \Gamma_{k}+\pi_{k-1}(C(G, \rho)) \stackrel{\mu}{\longrightarrow} \Re^{n-1}(G, \rho) \rightarrow \cdots$ is exact, where the map $\mu$ is defined in [5].

Theorem 1.3 (Rothenberg). (i) If $n$ is even,

$$
R^{n}(G, \rho) \otimes \mathrm{C} \cong R_{n, m} \oplus \pi_{k-1}(O(n-k)) \otimes \mathbf{C},
$$

where $R_{n, m} \subset \mathrm{C}\left[Z_{m}\right]$ the complex group ring, is the subspace generated by $\left\{g^{i}+(-1)^{n} g^{m-i}\right\}(i=1,2, \ldots,[m / 2]) ;$ and

(ii) $\mu \otimes \mathrm{C} \cong \Delta \oplus \psi \otimes \mathrm{C}$, where $\mu$ is the map in Theorem 1.2, and $\psi$ : $\pi_{k-1}\left(C\left(Z_{m}, \rho\right)\right) \rightarrow \pi_{k-1}(O(n-k))$ is the map induced by the inclusion with $\Delta$ defined as follows. For $f: S^{k-1} \rightarrow C\left(Z_{m}, \rho\right)$, let $\eta$ be the vector bundle over $S^{k}$ with $f$ as characteristic map. Let $Z_{m}$ act on $\eta$ via $\rho$. Then $\Delta(f)$ $=\sum_{j=1}^{m} \sigma\left(S(\eta), g^{j}\right) g^{j}$, where $\sigma$ is the Atiyah-Singer invariant [1].

These results were also proved by Browder and Petrie (which were announced in [3]) in the special case where the equivariant normal bundles are equivariantly trivial. The general case appeared in unpublished work of Rothenberg.

Remark 1.4. The rank $R_{n, m}$, which has been computed in [7], is given as follows:

$$
\text { rank } R_{n, m}= \begin{cases}(m-1) / 2 & \text { if } m \text { is odd, } \\ m / 2 & \text { if } m \text { is even and } n \text { is even, } \\ (m-2) / 2 & \text { if } m \text { is even and } n \text { is odd. }\end{cases}
$$

2. Computation of determinants. In this section we show how to compute the determinants of some matrices which will be useful in $\$ 3$.

Let $m>2$ and $x$ be positive integers such that $(x, m)=1$. We define $a_{m}(x)$ by $0<a_{m}(x)<m / 2$ and $a_{m}(x) \pm x \equiv 0(\bmod m)$ if $m \neq 0(\bmod 4)$, and $0<a_{m}(x)<m / 4$ and $\alpha_{m}(x) \pm x \equiv 0(\bmod m / 2)$ if $m \equiv 0(\bmod 4)$. It is easy to see that $\alpha_{m}(x)$ is well defined. Let

$$
\Lambda(m)= \begin{cases}\{k \mid 0<k<m / 2 \text { and }(k, m)=1\} \text { if } m \neq 0(\bmod 4), \\ \{k \mid 0<k<m / 4 \text { and }(k, m)=1\} \text { if } m \equiv 0 \quad(\bmod 4) .\end{cases}
$$

Proposition 2.1. $\Lambda(m)$ is an abelian group with multiplication $x \circ y=$ $\alpha_{m}(x y)$.

Proof. Note that $(m, m-1)=1$. If $m \neq 0(\bmod 4), \Lambda(m)$ with the multi- 
plication $a_{m}(x y)$ may be identified in the obvious way with $Z_{m}^{\times} /\{1, m-1\}$, where $Z_{m}^{X}$ is the group of units of $Z_{m}$. If $m \equiv 0(\bmod 4)$, it is easy to see that $(m, m / 2-1)=(m, m / 2+1)=1 . \Lambda(m)$ with the multiplication $\alpha_{m}(x y)$ may be identified with $Z_{m}^{\times} /\{1, m / 2-1, m / 2+1, m-1\}$. In either case, $a_{m}$ : $Z_{m}^{\times} \rightarrow \Lambda(m)$ is the projection map.

By the fundamental theorem of finite abelian groups, there exist cyclic subgroups $\Lambda_{j}(m)(j=1, \ldots, s)$ of $\Lambda(m)$ such that $\Lambda(m)$ is a direct product $\Pi_{j=1}^{s} \Lambda_{j}(m)$. Let $e_{j} \in \Lambda_{j}(m)$ be generators that are fixed once and for all. Let $t_{j}$ be the order of $\Lambda_{j}(m)$. For each $x \in \Lambda(m)$, there are well defined $\phi_{j}(x) \epsilon$ $Z_{t_{j}}$ such that $x=\alpha_{m}\left(\Pi_{j=1}^{s} e_{j}^{\phi_{j}(x)}\right)$. The following lemma is obvious.

Lemma 2.2. For $x, y \in \Lambda(m), \phi_{j}\left(\alpha_{m}(x y)\right) \equiv \phi_{j}(x)+\phi_{j}(y)\left(\bmod t_{j}\right)$.

Let $r$ be a positive integer, which will be fixed throughout this section; and let $x$ be a positive integer such that $(x, m)=1$. We define $\delta_{m}(x)$ as follows:

Case i. $m \neq 0(\bmod 4)$,

$$
\delta_{m}(x) \equiv \begin{cases}0(\bmod 2) & \text { if } x-[x / m] m<m / 2 \\ r(\bmod 2) & \text { otherwise }\end{cases}
$$

Case ii. $m \equiv 0(\bmod 4)$,

$$
\delta_{m}(x) \equiv\left\{\begin{array}{lll}
0 & (\bmod 2) & \text { if } 0<x-[x / m] m<m / 4 \\
r-1(\bmod 2) & \text { if } m / 4<x-[x / m] m<m / 2 \\
1 & (\bmod 2) & \text { if } m / 2<x-[x / m]_{m}<3 m / 4 \\
r & (\bmod 2) & \text { if } 3 m / 4<x-[x / m] m<m .
\end{array}\right.
$$

From now on, in order to simplify notation, we shall write $\Lambda=\Lambda(m)$, $\alpha(x)=a_{m}(x)$, etc., when $m$ is understood.

Lemma 2.3. For positive integers $a, b$ such that $(a, m)=(b, m)=1$, $\delta(a a(b)) \equiv \delta(a b)+\delta(b)(\bmod 2)$.

Proof. Case i. $m \neq \equiv(\bmod 4)$. Observe that $a(x) \equiv x$ or $-x(\bmod m)$ according as $\delta(x)=0(\bmod 2)$, or $r(\bmod 2)$, respectively. Thus $a \alpha(b) \equiv a b$ or $-a b(\bmod m)$ according as $\delta(b)=0(\bmod 2)$ or $r(\bmod 2)$, respectively. The lemma is now clear.

Case ii. $m \equiv 0(\bmod 4)$. The same method applies in this case. $\square$

Let $\left\{\Lambda_{j}\right\}, j=1, \ldots, t$, be the maximal family of factors of $\Lambda=\Pi_{j=1}^{s} \Lambda_{j}$ that have the property that either $t_{j} \equiv 0(\bmod 4)$ or $\delta\left(e_{j}^{t_{j}}\right) \equiv 0(\bmod 2)$. Let $\Lambda^{*}=\Pi_{j=1}^{t} \Lambda_{j}$. We will define a map $\psi: \Lambda^{*} \rightarrow Z_{4}$ as follows: For $j=1, \ldots$, $t$, let $\psi\left(e_{j}\right)$ be a solution, which will be fixed once and for all, of 


$$
t_{j} \psi\left(e_{j}\right) \equiv 2 \delta\left(e_{j}^{t_{j}^{j}}\right) \quad(\bmod 4) .
$$

Then define

$$
\psi\left(\alpha\left(e_{j}^{k}\right)\right) \equiv k \psi\left(e_{j}\right)+2 \delta\left(e_{j}^{k}\right) \quad(\bmod 4) .
$$

Lemma 2.6. $\psi: \Lambda_{j} \rightarrow Z_{4}$ is well defined.

Proof. Since $\alpha\left(e_{j}^{t_{j}+k}\right)=\alpha\left(e_{j}^{k}\right)$,

$$
\begin{aligned}
\psi\left(a\left(e_{j}^{t_{j}+k}\right)\right) & \equiv\left(k+t_{j}\right) \psi\left(e_{j}\right)+2 \delta\left(e_{j}^{t_{j}+k}\right)(\bmod 4), \\
& \equiv k \psi\left(e_{j}\right)+2 \delta\left(e_{j}^{t}\right)+2 \delta\left(e_{j}^{t_{j}+k}\right) \quad(\bmod 4)
\end{aligned}
$$

by (2.4),

$$
\equiv k \psi\left(e_{j}\right)+2 \delta\left(e_{j}^{k} \alpha\left(e_{j}^{t} j\right)\right)(\bmod 4)
$$

by Lemma 2.3,

$$
\begin{aligned}
& \equiv k \psi\left(e_{j}\right)+2 \delta\left(e_{j}^{k}\right)(\bmod 4), \\
& \equiv \psi\left(\alpha\left(e_{j}^{k}\right)\right)(\bmod 4),
\end{aligned}
$$

as required. $\square$

$$
\text { For } x \in \Lambda^{*}, x=\alpha\left(\Pi_{j=1}^{t} x_{j}\right), x_{j} \in \Lambda_{j} \text {. Define }
$$

$$
\psi(x) \equiv \sum_{j=1}^{t} \psi\left(x_{j}\right)+2 \delta\left(\prod_{j=1}^{t} x_{j}\right) \quad(\bmod 4)
$$

It is obvious that $\psi: \Lambda^{*} \rightarrow Z_{4}$ is well defined.

Lemma 2.8. For $j=1, \ldots, t$,

$$
\psi\left(\alpha\left(e_{j}^{p+q}\right)\right) \equiv \psi\left(\alpha\left(e_{j}^{p}\right)\right)+\psi\left(\alpha\left(e_{j}^{q}\right)\right)+2 \delta\left(\alpha\left(e_{j}^{p}\right) \alpha\left(e_{j}^{q}\right)\right)(\bmod 4) .
$$

Proof. By (2.5),

$$
\begin{aligned}
\psi\left(\alpha\left(e_{j}^{p+q}\right)\right) & \equiv(p+q) \psi\left(e_{j}\right)+2 \delta\left(e_{j}^{p+q}\right) \quad(\bmod 4), \\
\psi\left(a\left(e_{j}^{p}\right)\right) & \equiv p \psi\left(e_{j}\right)+2 \delta\left(e_{j}^{p}\right) \quad(\bmod 4), \\
\psi\left(\alpha\left(e_{j}^{q}\right)\right) & \equiv q \psi\left(e_{j}\right)+2 \delta\left(e_{j}^{q}\right) \quad(\bmod 4) .
\end{aligned}
$$

Therefore 


$$
\begin{aligned}
\psi\left(\alpha\left(e_{j}^{p+q}\right)\right)-\left(\psi\left(\alpha\left(e_{j}^{p}\right)\right)+\psi\left(\alpha\left(e_{j}^{q}\right)\right)\right) & \equiv 2 \delta\left(e_{j}^{p}\right)+2 \delta\left(e_{j}^{q}\right)+2 \delta\left(e_{j}^{p+q}\right)(\bmod 4), \\
& \equiv 2 \delta\left(e_{j}^{p}\right)+2 \delta\left(e_{j}^{p} \alpha\left(e_{j}^{q}\right)\right)(\bmod 4)
\end{aligned}
$$

by Lemma 2.3 ,

$$
\equiv 2 \delta\left(\alpha\left(e_{j}^{p}\right) \alpha\left(e_{j}^{q}\right)\right) \quad(\bmod 4)
$$

by Lemma 2.3 again, as required.

Proposition 2.9. For $x, y \in \Lambda^{*}, \psi(\alpha(x y)) \equiv \psi(x)+\psi(y)+2 \delta(x y)(\bmod 4)$.

Proof. Let $x=a\left(\Pi_{j=1}^{t} x_{j}\right), y=a\left(\Pi_{j=1}^{t} y_{j}\right)$. Then

$$
\begin{aligned}
\psi(\alpha(x y)) & =\psi\left(\alpha\left(\prod_{j=1}^{t} x_{j} y_{j}\right)\right) \\
& \equiv \sum_{j=1}^{t} \psi\left(\alpha\left(x_{j} y_{j}\right)\right)+2 \delta\left(\prod_{j=1}^{t} \alpha\left(x_{j} y_{j}\right)\right)(\bmod 4)
\end{aligned}
$$

by (2.7),

$$
\equiv \sum_{j=1}^{t}\left(\psi\left(x_{j}\right)+\psi\left(y_{j}\right)+2 \delta\left(x_{j} y_{j}\right)\right)+2 \delta\left(\prod_{j=1}^{t} \alpha\left(x_{j} y_{j}\right)\right)(\bmod 4)
$$

by Lemma 2.8; while

$$
\psi(x)+\psi(y) \equiv \sum_{j=1}^{t}\left(\psi\left(x_{j}\right)+\psi\left(y_{j}\right)\right)+2 \delta\left(\prod_{j=1}^{t} x_{j}\right)+2 \delta\left(\prod_{j=1}^{t} y_{j}\right)(\bmod 4)
$$

Consequently,

$$
\begin{aligned}
& \psi(\alpha(x y))-(\psi(x)+\psi(y)) \\
& \equiv 2 \sum_{j=1}^{t} \delta\left(x_{j} y_{j}\right)+2 \delta\left(\prod_{j=1}^{t} \alpha\left(x_{j} y_{j}\right)\right)+2 \delta\left(\prod_{j=1}^{t} x_{j}\right)+2 \delta\left(\prod_{j=1}^{t} y_{j}\right)(\bmod 4) .
\end{aligned}
$$

By Lemma 2.3,

$$
\begin{aligned}
2 \delta\left(\prod_{j=1}^{t} a\left(x_{j} y_{j}\right)\right) & =2 \delta\left(\left(\prod_{j=2}^{t} a\left(x_{j} y_{j}\right) a\left(x_{1} y_{1}\right)\right)\right) \\
& \equiv 2 \delta\left(\left(x_{1} y_{1} \prod_{j=2}^{t} \alpha\left(x_{j} y_{j}\right)\right)+\delta\left(x_{1} y_{1}\right)\right)(\bmod 4) ;
\end{aligned}
$$


and by successive applications of Lemma 2.3,

$$
\equiv 2 \delta\left(\prod_{j=1}^{t} x_{j} y_{j}\right)+2 \sum_{j=1}^{t} \delta\left(x_{j} y_{j}\right)(\bmod 4) .
$$

Thus

$$
\begin{aligned}
\psi(\alpha(x y))-(\psi(x)+\psi(y)) & \equiv 2 \delta\left(\prod_{j=1}^{t} x_{j}\right)+2 \delta\left(\prod_{j=1}^{t} y_{j}\right)+2 \delta\left(\prod_{j=1}^{t} x_{j} y_{j}\right)(\bmod 4) \\
& \equiv 2 \delta\left(\prod_{j=1}^{t} x_{j}\right)+2 \delta\left(\prod_{j=1}^{t} x_{j} a\left(\prod_{j=1}^{t} y_{j}\right)\right)(\bmod 4)
\end{aligned}
$$

by Lemma 2.3 ,

$$
\equiv 2 \delta\left(\alpha\left(\prod_{j=1}^{t} x_{j}\right) a\left(\prod_{j=1}^{t} y_{j}\right)\right)(\bmod 4)
$$

by Lemma 2.3 again,

$$
\equiv 2 \delta(x y)
$$

as required.

Theorem 2.10. For each $p \in \Lambda^{*}$, let $a_{p}$ be any complex number corre. sponding to $p$, then

$$
\operatorname{det}\left((-1)^{\delta(h k)} a_{a(b k)}\right)_{h, k \in \Lambda^{*}}= \pm \prod_{p \in \Lambda^{*}}\left(\sum_{q \in \Lambda^{*}} a_{q}{ }^{i} \psi(q)\left(\prod_{j=1}^{t} \omega_{j}^{\phi_{j}(p) \phi_{j}(q)}\right)\right),
$$

where $\omega_{j}$ is a primitive $t_{j}$ th root of unity.

Proof. Let $r(h)$ be defined by the equation $a(h r(h))=1$. Regard the $a_{p}$ 's as indeterminants, and let

$$
A=\left((-1)^{\delta(h k)} a_{a(h k)}\right)_{h, k \in \Lambda^{*}, k \neq 1^{\bullet}}
$$

Fix $p \in \Lambda^{*}$, and add ${ }_{i} \psi(q)\left(\Pi_{j=1}^{t} \omega_{j}^{\left.\phi_{j}(p) \phi_{j}(q)\right)}\right.$ times the qth column to the first column. Then 
$\operatorname{det}\left((-1)^{\delta(h k)} a_{\alpha(h k)}\right)_{h, k \in \Lambda^{*}}$

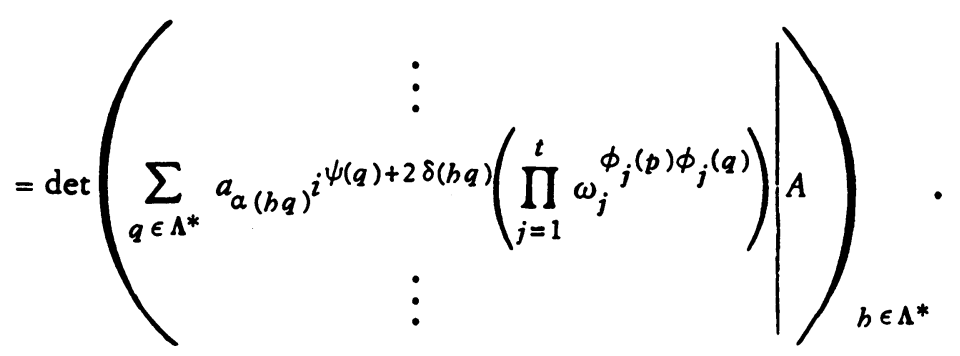

We now compute

$$
\begin{aligned}
& \sum_{q \in \Lambda^{*}} a_{a(h q)^{i}} \psi(q)+2 \delta(h q)\left(\prod_{j=1}^{t} \omega_{j}^{\phi_{j}(p) \phi_{j}(q)}\right) \\
& =i^{\psi(\tau(h))}\left(\prod_{j=1}^{t} \omega_{j}^{\phi_{j}(\tau(h)) \phi_{j}(p)}\right)\left(\sum_{q \in \Lambda^{*}} a_{a(h q)^{i}} \psi(a(h q))\left(\prod_{j=1}^{t} \omega_{j}^{\phi_{j}(p) \phi_{j}(a(h q))}\right)\right) \\
& =i^{\psi(\tau(h))}\left(\prod_{j=1}^{t} \omega_{j}^{\phi_{j}(\tau(h)) \phi_{j}(p)}\right)\left(\sum_{q \in \Lambda^{*}} a_{q}{ }^{i} \psi(q)\left(\prod_{j=1}^{t} \omega_{j}^{\left.\phi_{j}(p) \phi_{j}(q)\right)}\right)\right) .
\end{aligned}
$$

Thus $\Sigma_{q \in \Lambda^{*}} a_{q^{i}} \psi(q)\left(\Pi_{j=1}^{t} \omega_{j}^{\phi_{j}(p) \phi_{j}(q)}\right)$ is a factor of $\operatorname{det}\left((-1)^{\delta(b k)} a_{a(h k)}\right)_{h, k \in \Lambda^{*}}$ It is easy to see that, in general, for $p^{\prime} \neq p$,

$$
\sum_{q \in \Lambda^{*}} a_{q} i^{i} \psi(q)\left(\prod_{j=1}^{t} \omega_{j}^{\phi_{j}(p) \phi_{j}(q)}\right) \neq \sum_{q \in \Lambda^{*}} a_{q}{ }^{i} \psi(q)\left(\prod_{j=1}^{t} \omega_{j}^{\phi_{j}\left(p^{\prime}\right) \phi_{j}(q)}\right) .
$$

Thus

$$
\operatorname{det}\left((-1)^{\delta(h k)} a_{a(h k)}\right)_{h, k \in \Lambda^{*}}=c \prod_{p \in \Lambda^{*}}\left(\sum_{q \in \Lambda^{*}} a_{q}{ }^{i} \psi(q)\left(\prod_{j=1}^{t} \omega_{j}^{\phi_{j}(p) \phi_{j}(q)}\right)\right)
$$

for some constant $c$, which is easily seen to be \pm 1 . $\square$

Let $\operatorname{Im}(x+i y)=y$, where $x$ and $y$ are real.

Proposition 2.11. Suppose for all $q \in \Lambda^{*}$, the $a_{q}$ are real. Then if $p \in \Lambda^{*}$,

$$
\begin{aligned}
& \operatorname{Im} \sum_{q \in \Lambda^{*}} a_{q}{ }^{i} \psi(q)\left(\prod_{j=1}^{t} \omega_{j}^{\phi_{j}(p) \phi_{j}(q)}\right) \\
& \quad=\sum_{q \in \Lambda^{*} ; q<\tau(q)}\left(a_{q}-(-1)^{\psi(\tau(q))} a_{\tau(q)}\right)\left(\operatorname{Im}\left({ }_{i} \psi(q) \prod_{j=1}^{t} \omega_{j}^{\phi_{j}(p) \phi_{j}(q)}\right)\right) .
\end{aligned}
$$


Proof. Let $A(q)=i^{\psi(q)} \Pi_{j=1}^{t} \omega_{j}^{\phi_{j}(p) \phi_{j}(q)}$, and let $\overline{A(q)}$ be the complex conjugate of $A(q)$. It is easy to check that $\overline{A(q)}=(-1)^{\psi(q)} A(r(q))$. Thus

$$
\begin{aligned}
\sum_{q \in \Lambda^{*}} a_{q} A(q)- & \sum_{q \in \Lambda^{*}} a_{q} \overline{A(q)} \\
= & \sum_{q<\tau(q)} a_{q} A(q)+\sum_{q<\tau(q)} a_{\tau(q)} A(\tau(q)) \\
& -\sum_{q<\tau(q)}(-1)^{\psi(q) a_{q} A(\tau(q))-} \sum_{q<\tau(q)}(-1)^{\psi(q)} a_{\tau(q)} A(q) \\
= & \sum_{q<\tau(q)} a_{q}\left(A(q)-(-1)^{\psi(q)} A(\tau(q))\right)-(-1)^{\psi(\tau(q)))} \\
& \cdot \sum_{q<\tau(q)} a_{\tau(q)}\left(A(q)-(-1)^{\psi(\tau(q))} A(\tau(q))\right) \\
= & \sum_{q<\tau(q)}\left(a_{q}-(-1)^{\psi(\tau(q))} a_{\tau(q)}\right)(A(q)-\overline{A(q))} .
\end{aligned}
$$

Remark 2.12. If $r$ is even, then $\delta(x) \equiv 0(\bmod 2)$ for all $x \in \Lambda$. Hence $\Lambda^{*}=\Lambda$, and $\psi(x)=0$ for all $x \in \Lambda$.

Remark 2.13. If $m$ is an odd prime, then $\Lambda$ is cyclic.

Remark 2.14. If $\Lambda$ is cyclic and $r$ is even, then

$$
\operatorname{det}\left(a_{a(h k)}\right)_{h, k \in \Lambda}= \pm \prod_{p=1}^{\lambda}\left(\sum_{q=1}^{\lambda} a_{q} \omega^{p q}\right)
$$

where $\lambda=|\Lambda|$ and $\omega$ is a primitive $\lambda_{\text {th }}$ root of unity.

The following example shows the necessity of restricting the definition of $\psi$ to $\Lambda^{*}$.

Example 2.15. If $m=17$ and $r$ is odd, there exists no map $\psi: \Lambda \rightarrow Z_{4}$ such that Proposition 2.9 holds.

Proof. Suppose there were $\psi: \Lambda \rightarrow Z_{4}$ such that Proposition 2.9 holds. Let $e \in \Lambda$ be a generator, then

$$
\sum_{x \in \Lambda} \psi(\alpha(x e)) \equiv \sum_{x \in \Lambda} \psi(x)+8 \psi(e)+2 \sum_{x \in \Lambda} \delta(x e)(\bmod 4)
$$

Consequently,

$$
\sum_{x \in \Lambda} \delta(x e) \equiv 0 \quad(\bmod 2)
$$


But

$$
\sum_{x \in \Lambda} \delta(x e) \equiv \sum_{t=0}^{7} \delta\left(\alpha\left(e^{t}\right) e\right) \equiv \sum_{t=0}^{7}\left(\delta\left(e^{t+1}\right)+\delta\left(e^{t}\right)\right) \equiv \delta\left(e^{8}\right) \quad(\bmod 2) .
$$

Thus $\delta\left(e^{8}\right) \equiv 0(\bmod 2)$. It can be easily checked that if $e$ is a generator of $\Lambda, e^{8} \equiv-1(\bmod 17)$. Hence $\delta\left(e^{8}\right) \equiv 1(\bmod 2)$. This contradiction demonstrates the nonexistence of $\psi$. $\square$

3. Coefficients in the $G$-signature formula. Let the functions $\Phi_{k}(\theta), \theta \epsilon$ $[0, \pi]$, be defined by the equation

$$
\prod_{\theta}=\prod_{j}\left(\frac{\tanh (i \theta / 2)}{\tanh \left(\left(x_{j}+i \theta\right) / 2\right)}\right)=\sum_{k} \Phi_{k}(\theta) c_{k}+\text { decomposable terms }
$$

where $c_{k}$ is the kth Chern class.

Lemma 3.1.

$$
\begin{gathered}
\Phi_{2 r-1}(\theta)=(-1)^{r} \frac{i}{\sin ^{2 r-1} \theta} P\left(\cos ^{2} \theta\right), \\
\Phi_{2 r}(\theta)=(-1)^{r+1} \frac{\cos \theta}{\sin ^{2 r} \theta} Q\left(\cos ^{2} \theta\right)
\end{gathered}
$$

where $P$ and $Q$ are some polynomials with nonnegative coefficients.

Proof. By a result of F. Hirzebruch [4],

$$
\begin{aligned}
\sum_{j=0}^{\infty}(-1)^{j} \Phi_{j}(\theta) z^{j} & =1-z \frac{d}{d z}\left(\frac{\tanh (i \theta / 2)}{\tanh ((z+i \theta) / 2)}\right) \\
& =1+z \operatorname{csch}(z+i \theta) .
\end{aligned}
$$

The lemma can now be proved easily by induction.

The following corollaries follow easily by elementary calculations.

Corollary 3.2. (i) $\Phi_{r}(\pi-\theta)=(-1)^{r-1} \Phi_{r}(\theta)$,

(ii) $\Phi_{r}(\pi+\theta)=-\Phi_{r}(\theta)$,

(iii) $\Phi_{r}(2 \pi-\theta)=(-1)^{r} \Phi_{r}(\theta)$.

Corollary 3.3. (i) $\Phi_{4 k}(\theta)$ is monotonic increasing on $[0, \pi]$;

(ii) $\Phi_{4 k+1}(\theta)_{i}$ is monotonic decreasing on $[0, \pi / 2]$, and monotonic increasing on $[\pi / 2, \pi]$;

(iii) $\Phi_{4 k+2}(\theta)$ is monotonic decreasing on $[0, \pi]$; 
(iv) $\Phi_{4 k+3}(\theta) i$ is monotonic increasing on $[0, \pi / 2]$, and monotonic decreasing on $[\pi / 2, \pi]$;

(v) $\left|\Phi_{r}(\theta)\right|$ is monotonic decreasing on $[0, \pi / 2]$ and monotonic increasing on $[\pi / 2, \pi]$.

Corollary 3.4. For each integer $m>2,\left|\Phi_{r}(2 \pi / m)\right|<2^{-r}(\sec (\pi / m))\left|\Phi_{r}(\pi / m)\right|$.

Let $m>2$ be an integer.

Corollary 3.5. There exist integers $s$ and $t$ such that $s \neq t, 0<s, t<$ $m / 2$, and $(s, m)=(t, m)=1$ and such that $\Phi_{r}(2 s \pi / m)=(-1)^{r-1} \Phi_{r}(2 t \pi / m)$ if and only if $m \equiv 0(\bmod 4)$.

Proof. It is easy to see that $2 s+2 t=m$, so $m$ is even. Since $(s, m)=$ $(t, m)=1, s, t$ must be odd. Hence $m \equiv 0(\bmod 4)$.

Conversely, suppose $m=4 k$, and choose $0<s<2 k$ such that $(s, m)=$ 1. Then $s$ is odd and $(s, k)=1$. There exist $p, q$ such that $p s+q k=1$. So $(2 p-q) k-p(2 k-s)=1$. Thus $((2 k-s), k)=1$. It is obvious that $2 k-s$ is odd, $2 k-s \neq s$, and $\Phi_{r}(2 s \pi / m)=(-1)^{r-1} \Phi_{r}\left(2(2 k-s)_{\pi} / m\right)$.

Consider the following matrix

$$
\widetilde{\Phi}_{r}=\left(\Phi_{r}\left(\frac{2 h k \pi}{m}\right)\right)_{h, k \in \Delta},
$$

where $\Lambda$ is defined in $\$ 2 . \tilde{\Phi}_{r}$ plays the dominant role in the G-signatures of cyclic group actions on homotopy sphere pairs.

For any positive integer $x$ such that $(m, x)=1$, let $a(x), \delta(x)$ be defined as in $\$ 2$. It is easy to see that

$$
\Phi_{r}\left(\frac{2 h k \pi}{m}\right)=(-1)^{\delta(h k)} \Phi_{r}\left(\frac{2 \alpha(b k) \pi}{m}\right)
$$

where $h, k \in \Lambda$. As a special case of Theorem 2.10 we have the following

Theorem 3.6. Let $\Lambda^{*}, \psi(q), \phi_{i}(q)$, and $\omega_{i}$ be defined as in $\S 2$. Then

$$
\operatorname{det}\left(\Phi_{r}\left(\frac{2 h k \pi}{m}\right)\right)_{h, k \in \Lambda^{*}}= \pm \prod_{p \in \Lambda^{*}}\left(\sum_{q \in \Lambda^{*}} \Phi_{r}\left(\frac{2 q \pi}{m}\right) i \psi(q)\left(\prod_{j=1}^{t} \omega_{j}^{\phi_{j}(p) \phi_{j}(q)}\right)\right) .
$$

Corollary 3.7. Let $\lambda=\left|\Lambda^{*}\right|$. If $r \geq \ln ((\lambda-1) \sec (\pi / m)) / \ln 2$, then

$$
\operatorname{det}\left(\Phi_{r}(2 h k \pi / m)\right)_{h, k \in \Lambda^{*}} \neq 0 .
$$

Proof. It is easy to see that if $b_{i}, \beta_{i}(i=0, \ldots, n)$ are complex num- 
bers such that $\left|b_{0}\right|>\sum_{j=1}^{n}\left|b_{j}\right|$ and $\left|\beta_{i}\right|=1(i=0, \ldots, n)$, then $\sum_{i=0}^{n} b_{i} \beta_{i}$ $\neq 0$. Thus Corollary 3.7 is an easy consequence of Corollary 3.4 and Theorem 3.6. $\square$

For small $m$ 's, we may compute directly to get

Corollary 3.8. $\operatorname{det}\left(\Phi_{r}(2 h k \pi / m)\right)_{h, k \in \Lambda^{*}} \neq 0$ for $m \leq 12$.

Remark 3.9. Based on these observations, it is reasonable to conjecture that $\operatorname{det}\left(\Phi_{r}(2 h k \pi / m)\right)_{h, k \in \Lambda^{*}} \neq 0$. In fact, Rothenberg conjectured in his talk at the Second Conference on Transformation Groups that det $\widetilde{\Phi}_{r} \neq 0$ for each odd prime $m$.

Remark 3.10. The first estimation of $r$ for a fixed odd prime $m$ for which det $\widetilde{\Phi}_{r} \neq 0$ was given by Schultz [8]. His method can also be applied for $m$ which are not necessarily odd primes. However, it appears to us that Schultz's method cannot be used to show that $\operatorname{det} \widetilde{\Phi}_{r} \neq 0$ for all $m$ and all $r$. Our approach is different from his and is, perhaps, a hopeful way to prove the conjecture.

4. The main results. For a positive integer $m \neq 2$, let $\Lambda^{\prime}=\{k \mid 0<k<$ $m / 2,(k, m)=1\}$. Let $\left(\Sigma^{2 n}, F^{2 r} ; Z_{m}\right)$ be a semifree $Z_{m}$ action on a homotopy sphere pair. The equivariant normal bundle $\nu$ to $F^{2 r}$ in $\Sigma^{2 n}$ splits equivariantly into a Whitney $\operatorname{sum} \nu=\bigoplus_{j \in \Lambda^{\prime}} \nu_{j}$ so that each factor $\nu_{j}$ is invariant under the $Z_{m}$ action, and the restriction of the action to each fiber of $\nu_{j}$ is just complex multiplication by $\exp (2 j \pi i / \mathrm{m})$. Let $g$ be a generator of $Z_{m}$. By the Atiyah-Singer $G$-signature Theorem $[1,6.12]$, we have

$$
\begin{aligned}
\operatorname{Sign} & \left(g^{h}, \Sigma^{2 n}\right) \\
= & 2^{r} \prod_{k \in \Lambda^{\prime}}(i \tan (b k \pi / m))^{-n} k \prod_{k \in \Lambda^{\prime}}\left(\sum_{j} \Phi_{j}\left(\frac{2 b k \pi}{m}\right) c_{j}\left(\nu_{k}\right)\right) \cap\left[F^{2 r}\right]
\end{aligned}
$$

for $h \in \Lambda^{\prime}$ and where $n_{k}=\operatorname{dim}_{C} \nu_{k}$. Note that since $F^{2 r}$ is a suspension, products of positive dimensional classes are zero. Hence

$$
\operatorname{Sign}\left(g^{h}, \Sigma^{2 n}\right)=K_{h}\left(\sum_{k \in \Lambda^{\prime}} \Phi_{r}\left(\frac{2 b k \pi}{m}\right) c_{r}\left(\nu_{k}\right)\right) \cap\left[F^{2 r}\right]
$$

where $h \in \Lambda^{\prime}$ and $K_{h}$ are constant depending only on $h$.

By definition, Sign $\left(g^{b}, \Sigma^{2 n}\right)$ is defined on the $Z_{m}$ module $H^{n}\left(\Sigma^{2 n}, Q\right)$, which is zero. So $\operatorname{Sign}\left(g^{h}, \Sigma^{2 n}\right)=0$ for all $h \in \Lambda^{\prime}$. If $m \neq 0(\bmod 4), \Lambda^{\prime}=$ $\Lambda$, where $\Lambda$ is as in $\S 2$. If det $\widetilde{\Phi}_{r} \neq 0$, where $\widetilde{\Phi}_{r}$ is as in $\S_{3}$, it is easy to see that $c_{r}\left(\nu_{k}\right)=0$ for all $k \in \Lambda$. Since the stable complex vector bundles 
over spheres are determined by their Chern classes, the $\nu_{j}$ are stably trivial as complex vector bundles. Thus we have proved the following

Theorem 4.1. For $m \neq 2$ and $m \neq 0(\bmod 4)$, let $\left(\Sigma^{2 n}, F^{2 r} ; Z_{m}\right)$ be a semifree $Z_{m}$ action on a homotopy spinere pair. If det $\widetilde{\Phi}_{r} \neq 0$, then the equivariant normal bundle $\nu$ to $F^{2 r}$ in $\Sigma^{2 n}$ is equivariantly stably trivial.

Remark 4.2. We say a $Z_{m}, m \neq 2$, equivariant bundle is trivial if each equivariant factor is trivial as a complex vector bundle.

Remark 4.3. This result has also been discovered and proved by Schultz [8] by a different argument.

Let $\rho: Z_{m} \rightarrow U(n-r)$ be a fixed unitary fixed point free representation of complex dimension $n-r$. If $m \neq 0(\bmod 4)$, and det $\widetilde{\Phi}_{r} \neq 0$, one sees, as an easy consequence of Theorem 4.1, that the image of $\gamma$ in Theorem 1.2 is $\Gamma_{2 r}$, a finite group. It has also been proved in [5] that $\mathbb{R}^{2 n}\left(Z_{m}, \rho\right)$ is finite. Hence we have

Theorem 4.4. For $m \neq 2$ and $m \neq 0(\bmod 4)$, let $\rho: Z_{m} \rightarrow U(n-r)$ be a fixed unitary fixed point free representation of complex dimension $n-r$. If $\operatorname{det} \widetilde{\Phi}_{r} \neq 0$, then $\mathcal{C}^{2 n}\left(Z_{m}, \rho\right)$ is finite.

As an easy consequence of Theorems 1.2, 1.3 and 4.4, we have

Corollary 4.5. Let $m, \rho$ be as in Theorem 4.4. If det $\tilde{\Phi}_{r} \neq 0$, then

$\operatorname{rank} \mathcal{C}^{2 n-1}\left(Z_{m}, \rho\right) \otimes \mathrm{C}=\operatorname{rank} R_{n, m}+\operatorname{rank} \pi_{2 r-1}(O(2 n-2 r)) \otimes \mathrm{C}$

$$
-\operatorname{rank} \pi_{2 r-1}\left(C\left(Z_{m}, \rho\right)\right) \otimes \mathbf{C},
$$

where rank $R_{n, m}$ is as in Remark 1.4; and $\rho=\Sigma_{j \in \mathbf{\Lambda}} n_{j} t^{j}$,

$$
\operatorname{rank} \pi_{2 r-1}\left(C\left(Z_{m}, \rho\right)\right) \otimes \mathbf{C}=\operatorname{Card}\left\{n_{j} \mid n_{j} \geq r\right\} \text {. }
$$

Remark 4.6. Corollary 3.7 shows that for fixed $m$ and $r$ even and sufficiently large, det $\widetilde{\Phi}_{r} \neq 0$.

In the rest of this section, we will consider the case $m \equiv 0(\bmod 4)$. Let $m=4 s$. If $n \geq 3 r$, we consider $\rho=n_{1} t+n_{2} t^{2 s-1}$ for $n_{1} \geq r_{1} n_{2} \geq r$. Then $C\left(Z_{m}, \rho\right) \cong U\left(n_{1}\right) \times U\left(n_{2}\right)$, and $\pi_{2 r-1}\left(U\left(n_{1}\right) \times U\left(n_{2}\right)\right)=Z \oplus Z$. The map $\Delta$ : $\pi_{2 r-1}\left(U\left(n_{1}\right) \times U\left(n_{2}\right)\right) \rightarrow \mathrm{C}\left[Z_{m}-1\right]$ in Theorem 1.3 can be computed explicitly as follows: $B y[1,6.15], \Delta\left(f_{1}, f_{2}\right)\left(g^{2 s}\right)=\operatorname{Sign}\left(\left(s^{2 r}\right)^{2}\right)=0$, and

$$
\Delta\left(f_{1}, f_{2}\right)\left(g^{h}\right)=K_{h}\left(\Phi_{r}\left(\frac{2 h \pi}{4 s}\right)\left(f_{1}^{*} c_{r}+(-1)^{r-1} f_{2}^{*} c_{r}\right)\right) \cap\left[s^{2 r}\right],
$$


where $h \neq 2 s$ and $f_{i}^{*} c_{r}$ is the Chern class of the complex vector bundle induced by the map $f_{i}: s^{2 r-1} \rightarrow U\left(n_{i}\right)(i=1,2)$. It is clear that $\operatorname{dim}_{C} \operatorname{ker} \Delta=$ 1. Note that if $r$ is odd, the map $\Psi$ in Theorem 1.3 vanishes. Hence we have, by Theorem 1.3,

Theorem 4.7. There exist infinitely many inequivalent semifree $Z_{4 s}$ actions on $S^{2 n}$ with fixed point set $S^{2 r}$ for $n \geq 3 r$ and $r$ odd.

If $r$ is even, $n \geq 5 r$ and $s \geq 3$, let $\rho=n_{1} t+n_{2} t^{s-1}+n_{3} t^{s+1}+n_{4} t^{2 s-1}$ for $n_{i} \geq r(i=1,2,3,4)$. A similar computation shows that $\operatorname{dim}_{\mathrm{C}} \operatorname{ker} \Delta=2$ and $\operatorname{dim}_{C}(\Psi \mid$ ker $\Delta)=1$. Hence we have, by Theorem 1.3,

Theorem 4.8. There exist infinitely many inequivalent semifree $Z_{45}$ actions on $S^{2 n}$ with fixed point set $S^{2 r}$ for $n \geq 5 r, s \geq 3$ and $r$ even.

Remark 4.9. This is the first known family of infinitely many semifree actions on even dimensional spheres. It should be noted that the previous attempt to prove this sort of result which was announced in [9] is false.

Remark 4.10. Let $\rho$ be the representation as in the proof of Theorem 4.7 (Theorem 4.8, respectively). By the proof of Theorem 4.7 (Theorem 4.8, respectively), $\operatorname{dim}_{C} \operatorname{ker} \mu \otimes C=1$. By Theorem $1.2, \operatorname{dim}_{C} \operatorname{Im} \gamma \otimes C=1$ where $\gamma: C^{2 n}\left(Z_{4 s}, \rho\right) \rightarrow \Gamma_{2 k}+\pi_{2 k-1}\left(C\left(Z_{4 s}, \rho\right)\right)$ is the classifying map of the equivariant normal bundles to the fixed point sets of semifree $Z_{4 s}$ actions on homotopy sphere pairs. Thus, in general, the equivariant normal bundle to the fixed point set of a semifree $Z_{4 s}$ action on a homotopy sphere pair is not equivariantly stably trivial. Nevertheless, we have the following:

Theorem 4.11. Let $\left(\Sigma^{2 n}, F^{2 r} ; Z_{4 s}\right)$ be a semifree $Z_{4 s}$ action on a homotopy sphere pair. If det $\widetilde{\Phi}_{r} \neq 0$, then the normal bundle of $F^{2 r}$ in $\Sigma^{2 n}$ is stably trivial as a complex (real, respectively) vector bundle if $r$ is odd (even, respectively), where $\widetilde{\Phi}_{r}$ is as in $\$ 3$.

Proof. Note that $\Phi_{r}(2 h(2 s-k) \pi / 4 s)=(-1)^{r} \Phi_{r}(2 h k \pi / 4 s)$ and $\Lambda^{\prime}=\{k \mid k \epsilon$ $\Lambda$ or $2 s-k \in \Lambda$, where $\Lambda$ is as in $\$ 2$. Observe that since $F^{2 r}$ is a homotopy sphere, the only nonzero classes are in dimension $2 r$, and, since $F^{2 r}$ is a suspension, products of positive dimensional classes are zero. Hence

$$
\begin{aligned}
\operatorname{Sign}\left(g^{h}, \Sigma^{2 n}\right) & =K_{h}\left(\sum_{k \in \Lambda^{\prime}} \Phi_{r}\left(\frac{2 h k \pi}{4 s}\right) c_{r}\left(\nu_{k}\right)\right) \cap\left[F^{2 r}\right] \\
& =K_{h}\left(\sum_{k \in \Lambda} \Phi_{r}\left(\frac{2 h k \pi}{4 s}\right) c_{r}\left(\nu_{k} \oplus(-1)^{r-1} \nu_{2 s-k}\right)\right) \cap\left[F^{2 r}\right] .
\end{aligned}
$$


By the argument used to prove Theorem 4.1, it follows that the direct sums $\nu_{k} \oplus(-1)^{r-1} \nu_{2 s-k}(k \in \Lambda)$ are stably trivial as complex vector bundles. If $r$ is odd,

$$
\nu=\bigoplus_{k \in \Lambda^{\prime}} \nu_{k}=\bigoplus_{k \in \mathbf{\Lambda}}\left(\nu_{k} \oplus \nu_{2 s-k}\right)
$$

Thus $\nu$ is stably trivial as a complex vector bundle. If $r$ is even, $\nu_{k}$ is stably equivalent to $\nu_{2 s-k}$, and

$$
\nu=\bigoplus_{k \in \Lambda^{\prime}} \nu_{k}=2 \underset{k \in \mathbf{\Lambda}}{\bigoplus} \nu_{k}
$$

Note that $\pi_{4 j}(0)=Z_{2}$ or 0 . Thus $\nu$ is stably trivial as a real vector bundle $\square$

Remark 4.12. The above argument also shows that for a semifree $Z_{4 s}$ action on a homotopy sphere pair $\left(\Sigma^{2 n}, F^{2 r}\right)$, with a local representation $\rho=$ $\Sigma_{j \in \Lambda^{\prime}} n_{j} t^{j}$ such that either $n_{j}<r$ or $n_{2 s-j}<r$ for $j \in \Lambda^{\prime}$, the equivariant normal bundle to $F^{2 r}$ is equivariantly stably trivial under the assumption $\operatorname{det} \widetilde{\Phi}_{r} \neq 0$.

Finally we can prove the following theorem by an argument similar to the one we used to prove Corollary 4.5.

Theorem 4.13. If det $\widetilde{\Phi}_{r} \neq 0$, then rank $C^{2 n}\left(Z_{4 s}, \rho\right) \otimes C=r(\rho)$ and $\operatorname{rank} \mathcal{C}^{2 n-1}\left(Z_{4 s}, \rho\right) \otimes \mathrm{C}=\operatorname{rank} R_{n, 4 s}+\operatorname{rank} \pi_{2 r-1}(O(2 n-2 r)) \otimes \mathrm{C}$ $-\operatorname{rank} \pi_{2 r-1}\left(C\left(Z_{4 s}, \rho\right)\right) \otimes C+r(\rho)$,

where

$$
r(\rho)=\operatorname{Card}\left\{n_{j} \mid n_{j} \geq r\right\}-\operatorname{Card}\left\{n_{j} \mid n_{j} \geq r, n_{2 s-j} \geq r\right\} .
$$

Remark 4.14. Again Corollary 3.7 shows for fixed $m$ and $r$ even and sufficiently large, det $\widetilde{\Phi}_{r} \neq 0$.

Remark 4.15. Our Theorems 4.1, 4.11 and Remark 4.10 to Theorems 4.7 and 4.8 settle Problem 1 for a large class of cases. Theorems 4.4, 4.7 and 4.8 do the same to Problem 2. Finally, Corollary 4.5 to Theorem 4.4 and Theorem 4.13 are related to Problem 3.

\section{REFERENCES}

1. M. F. Atiyah and I. M. Singer, The index of elliptic operators. III, Ann. of Math. (2) 87 (1968), 546-604. MR 38 \#5245.

2. W. Browder, Surgery and the theory of differentiable transformation groups, Proc. Conf. on Transformation Groups (New Orleans, La., 1967), Springer, New York, 1968, pp. 1-46. MR $41 \# 6242$.

3. W. Browder and T. Petrie, Diffeomorphisms of manifolds and semifree actions 
on homotopy spheres, Bull. Amer. Math. Soc. 77 (1971), 160-163. MR 42 \#8513.

4. F. Hirzebruch, Neue topologische Methoden in der algebraischen Geometrie, Ergebnisse der Mathematik und ihrer Grenzgebiete, Heft 9, Springer-Verlag, Berlin, 1956; English transl., Die Grundlehren der math. Wissenschaften, Band 131, SpringerVerlag, New York, 1966. MR 18, 509; 34 \#2573.

5. M. Rothenberg, Differentiable group actions on shperes, Proc. Adv. Study Inst. Algebraic Topology, vol. 2, Aarhus University, 1970, pp. 455-475.

6. M. Rothenberg and J. Sondow, Non-linear smooth representations of compact Lie groups (preprint).

7. T. Petrie, The Atiyah-Singer invariant, the Wall groups $L_{n}(\pi, 1)$, and the function $\left(t e^{x}+1\right) /\left(t e^{x}-1\right)$, Ann. of Math. (2) 92 (1970), 174-187. MR $47 \# 7761$.

8. R. Schultz, Rational h-cobordism invariants for lens space bundles, Quart. J. Math. Oxford Ser. (2) 25 (1974), 497-511.

9. K. Wang, Differentiable actions on $2 n$-spheres, Bull. Amer. Math. Soc. 78 (1972), 971-973. MR $46 \# 8252$.

DEPARTMENT OF MATHEMATICS, STATE UNIVERSITY OF NEW YORK AT BUFFALO, AMHERST, NEW YORK 14226 